\title{
Left Ventricular Function Assessment Using 2 Different Cadmium-Zinc-Telluride Cameras Compared with a $\gamma$-Camera with Cardiofocal Collimators: Dynamic Cardiac Phantom Study and Clinical Validation
}

\author{
Alban Bailliez ${ }^{1-3}$, Olivier Lairez ${ }^{4}$, Charles Merlin ${ }^{5}$, Nicolas Piriou ${ }^{6}$, Damien Legallois ${ }^{3,7}$, Tanguy Blaire ${ }^{1-3}$, \\ Denis Agostini ${ }^{3,8}$, Frederic Valette ${ }^{6}$, and Alain Manrique ${ }^{3,8}$ \\ ${ }^{I}$ Nuclear Medicine, IRIS, Polyclinique du Bois, Lille, France; ${ }^{2}$ Nuclear Medicine, UF 5881, Groupement des Hôpitaux de l'Institut \\ Catholique de Lille, Lomme, France; ${ }^{3}$ Normandie Université-EA4650, Caen, France; ${ }^{4}$ Nuclear Medicine, CHU de Toulouse, \\ Toulouse, France; ${ }^{5}$ Nuclear Medicine, Centre Jean Perrin, Clermont-Ferrand, France; ${ }^{6}$ Nuclear Medicine, CHU de Nantes, Nantes, \\ France; ${ }^{7}$ Cardiology, CHU de Caen, Caen, France; and ${ }^{8}$ Nuclear Medicine, CHU de Caen, Caen, France
}

This study compared two SPECT cameras with cadmium-zinc-telluride (CZT) detectors to a conventional Anger camera with cardiofocal collimators for the assessment of left ventricular (LV) function in a phantom and patients. Methods: A gated dynamic cardiac phantom was used. Eighteen acquisitions were processed on each CZT camera and the conventional camera. The total number of counts within a myocardial volume of interest varied from $0.25 \mathrm{kcts}$ to $1.5 \mathrm{Mcts}$. Ejection fraction was set to $33 \%, 45 \%$, or $60 \%$. Volume, LV ejection fraction (LVEF), regional wall thickening, and motion (17-segment model) were assessed. One hundred twenty patients with a low pretest likelihood of coronary artery disease and normal findings on stress perfusion SPECT were retrospectively analyzed to provide the reference limits for end-diastolic volume (EDV), end-systolic volume (ESV), ejection fraction, and regional function for each camera model. Results: In the phantom study, for each ejection fraction value, volume was higher for the CZT cameras than for the conventional camera, resulting in a decreased but more accurate LVEF (all $P<$ 0.001). In clinical data, body-surface-indexed EDV and ESV $\left(\mathrm{mL} / \mathrm{m}^{2}\right)$ were higher for one of the CZT cameras (Discovery NM 530c) than for the other (D-SPECT) or the conventional camera (respectively, $40.5 \pm 9.2$, $37 \pm 7.9$, and $35.8 \pm 6.8$ for EDV $[P<0.001]$ and $12.5 \pm 5.3,9.4 \pm 4.2$, and $8.3 \pm 4.4$ for ESV $[P<0.001]$ ), resulting in a significantly decreased LVEF: $70.3 \% \pm 9.1 \%$ vs. $75.2 \% \pm 8.1 \%$ vs. $77.8 \% \pm 9.3 \%$, respectively $(P<0.001)$. Conclusion: The new CZT cameras yielded global LV function results different from those yielded by the conventional camera. LV volume was higher for the Discovery NM 530c than for the D-SPECT or the conventional camera, leading to decreased LVEF in healthy subjects. These differences should be considered in clinical practice and warrant the collection of a specific reference database.

Key Words: CZT; myocardial perfusion imaging; left ventricular function; wall thickening; dynamic phantom; cardiofocal collimators

J Nucl Med 2016; 57:1370-1375

DOI: 10.2967/jnumed.115.168575

Received Oct. 20, 2015; revision accepted Mar. 26, 2016.

For correspondence or reprints contact: Alban Bailliez, Nuclear Medicine Department IRIS, 144 Avenues de Dunkerque, Polyclinique du Bois, 59000 Lille, France.

E-mail: abailliez@gmail.com

Published online Apr. 28, 2016.

COPYRIGHT (c) 2016 by the Society of Nuclear Medicine and Molecular Imaging, Inc.
$\mathbf{M}$

yocardial perfusion imaging is extensively used in patients with known or suspected coronary artery disease. In addition, the measurement of left ventricular (LV) ejection fraction (LVEF), enddiastolic volume (EDV) and end-systolic volume (ESV) using SPECT has been widely validated in comparison to other imaging techniques $(1,2)$ and is commonly used for prognosis assessment and clinical decision making (3). Moreover, it has been demonstrated that the reference limits of LV function are sex-specific but do not depend on type of tracer or acquisition camera when conventional Anger cameras are used (4), suggesting equivalency for patient management.

Dedicated cadmium-zinc-telluride (CZT) $\gamma$-cameras revealed a new step in SPECT myocardial perfusion imaging. These cameras allow for reduced imaging time (5) and reduced exposure of patients to radiation $(6,7)$ but are not equivalent in terms of count sensitivity and spatial resolution, leading to images with differences in sharpness and contrast-to-noise ratio in clinical practice (8). Previous publications have validated the performance and results of these cameras versus the conventional Anger camera in clinical practice $(9,10)$, but recent data demonstrated that when quantitative gated SPECT is performed using CZT cameras instead of Anger cameras, LV volume is overestimated, leading to a lower estimated LVEF in healthy patients (11). This difference is likely related to the increased spatial resolution, a condition that also significantly affects the assessment of segmental wall motion and thickening $(12,13)$. Alternatively, the multifocal collimators of IQ-SPECT technology (Siemens Healthcare) can be plugged into multipurpose cameras, offering better sensitivity and contrast-tonoise ratio than conventional Anger cameras equipped with lowenergy, high-resolution collimators (14).

Because commercially available CZT cameras and IQ-SPECT cameras have differences in sensitivity and spatial resolution, it remains unclear whether the type of camera may affect assessment of LV volume and LVEF. Consequently, the aim of this study was, first, to perform a head-to-head comparison of two commercially available CZT cameras versus a conventional Anger camera with IQ-SPECT collimators in the assessment of LV function on a dynamic cardiac phantom and, second, to confirm the phantom results by retrospectively analyzing data from patients with a low pretest likelihood of coronary artery disease. 


\section{MATERIALS AND METHODS}

\section{y-Cameras and Collimation Systems}

The first CZT camera was a Discovery NM 530c (DNM) (GE Healthcare) equipped with a multiple-pinhole collimator and 19 stationary CZT detectors that simultaneously image 19 cardiac views, with each detector comprising pixelated 5-mm-thick elements (matrix, $70 \times 70$; pixel size, $2.46 \times 2.46 \mathrm{~mm})(15)$. The second CZT camera was a D-SPECT (Biosensors International) operating with 9 mobile blocks of pixelated CZT detectors (pixel size, $2.46 \times 2.46 \mathrm{~mm}$ ) associated with a wide-angle square-hole tungsten collimator, with each block recording a total of 120 projections (16). The IQ-SPECT camera was a Symbia IQ-SPECT (IQ) (Siemens Medical Solutions) applying a high-sensitivity astigmatic convergent-geometry collimator for the image center and parallel holes for the edges connected to the camera, using a circular rotation $(28-\mathrm{cm}$ radius) centered on the heart $(128 \times 128$ matrix) (14). All SPECT datasets (phantom and patients) were acquired and reconstructed using the parameters currently recommended for clinical routine by each manufacturer, leading to a reconstructed pixel size of $4 \times 4 \times 4 \mathrm{~mm}, 4.92 \times 4.92 \times 4.92 \mathrm{~mm}$, and $4.8 \times 4.8 \times 4.8 \mathrm{~mm}$ for the DNM, D-SPECT, and IQ, respectively. No attenuation correction was performed.

\section{Dynamic Phantom Study}

We used the Amsterdam gated (AGATE) dynamic phantom (Vanderwilt Techniques) as a reference for volume and LVEF measurements (17). This phantom is a realistic 3-dimensional waterfilled torso that has 2 thin membranes simulating endocardial and epicardial walls with known ventricular volume and ejection fraction (Fig. 1). The lumen between these membranes was filled with a pertechnetate solution $(50 \mathrm{kBq} / \mathrm{mL})$ simulating a myocardial wall. The cardiac phantom stroke volume is controlled by a programmable adjustable pumping system, and an electrocardiography-triggered signal is produced at a constant heart rate. Eighteen acquisitions were obtained on each of the 3 cameras, with varying count-stop conditions of $0.25 \mathrm{kcts}, 0.5 \mathrm{kcts}, 0.75 \mathrm{kcts}, 1 \mathrm{Mcts}, 1.25 \mathrm{Mcts}$, and $1.5 \mathrm{Mcts}$ within a myocardial volume of interest. LVEF was set by the adjust-

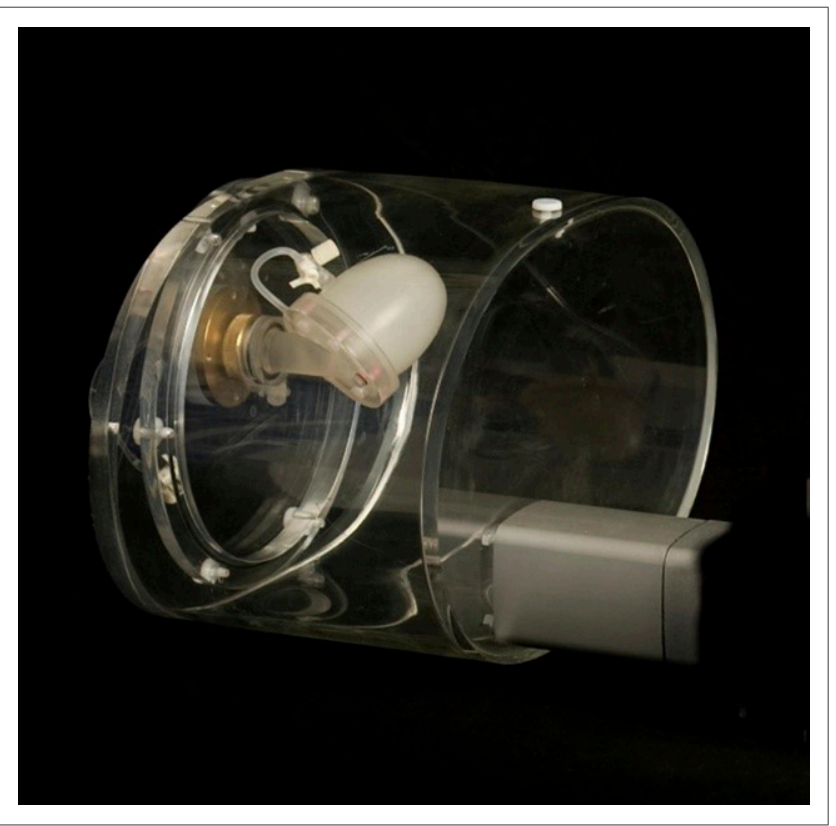

FIGURE 1. AGATE dynamic phantom. able pumping system (17) to $33 \%$ and $45 \%$ to mimic LV dysfunction or to $60 \%$ to mimic normal $\mathrm{LV}$ function. The acquisition parameters were as follows: a $70 \times 70$ matrix for the DNM camera; a $64 \times 64$ matrix for the D-SPECT camera, with a total of 120 projections recorded by each block in the heart area, which was defined on a short prescan acquisition (18); and a $128 \times 128$ matrix with 30 projections over $180^{\circ}$ and $30 \mathrm{~s}$ per projection for the IQ camera. The energy window was $140 \mathrm{keV} \pm 10 \%$ for all cameras.

\section{Clinical Data}

We retrospectively analyzed poststress acquisitions from 120 consecutive patients who had a low pretest likelihood of coronary artery disease and normal results on stress-only perfusion SPECT and were referred to our nuclear medicine departments for a routine evaluation of myocardial perfusion. Image datasets were collected at Clinique du Bois $(n=40)$ for the Discovery NM, at Centre Hospitalier Universitaire de Caen $(n=20)$ and Centre Jean Perrin $(n=20)$ for the D-SPECT, and at Centre Hospitalier Universitaire de Nantes $(n=$ $20)$ and Centre Hospitalier Universitaire Rangueil $(n=20)$ for the IQ. DICOM-stored datasets from 10 men and 10 women were retrieved for each camera and for both ${ }^{99 \mathrm{~m}} \mathrm{Tc}$-sestamibi and ${ }^{99 \mathrm{~m}} \mathrm{Tc}$-tetrofosmin to determine the reference limits for EDV, ESV, and LVEF, regional motion, and thickening for each camera model. For each camera, we used the reconstruction parameters recommended by its vendor. For the DNM, a Butterworth postprocessing filter (frequency, 0.37; order, 7) was applied to the reconstructed axial slices, which were subsequently reformatted in the standard cardiac axis for analysis. For the D-SPECT, a specific algorithm for iterative reconstruction (4 iterations) was used to compensate for the collimator-related loss of spatial resolution (16). For the IQ, images were routinely reconstructed using a 3-dimensional iterative algorithm (6 iterations, 4 subsets) with correction for the geometry of the astigmatic collimator (Flash 3D) and a postreconstruction filter (gaussian, $8.4 \mathrm{~mm}$ in full width at half maximum). EDV and ESV were indexed to body area for each patient, and the patients' characteristics were retrieved from hospital records. All reconstructed datasets were stored in DICOM format and subsequently processed by the QGS package (version 2008; Cedars Sinai) running on a Spectrum Dynamics workstation and using a 17-segment model for regional myocardial wall thickening and motion analysis (19). All images were anonymized. Our ethics committee (Comité de Protection des Personnes Nord-Ouest III) approved this retrospective study and waived the requirement to obtain informed consent.

\section{Statistical Analysis}

Continuous variables are presented as mean \pm SD. The ShapiroWilk test was used to assess the normality of continuous variables. We used multivariate ANOVA with post hoc comparison of means (Tukey test) or the Kruskal-Wallis test when appropriate for global comparison among the 3 cameras. Reproducibility among the Discovery NM, D-SPECT, and IQ for the measurement of EDV, ESV, and LVEF using the AGATE phantom was tested using 1-way random intraclass correlation coefficients (20). All statistical analyses were performed using $\mathrm{R}$ software (version 3.1.2; R Foundation for Statistical Computing). A $P$ value of less than 0.05 was considered statistically significant.

\section{RESULTS}

\section{Phantom Study}

With the AGATE phantom, the overall mean EDV, ESV, and LVEF for the DNM, D-SPECT, and IQ are shown in Figure 2. Measured phantom volume was higher and ejection fraction lower for the DNM than for the D-SPECT or IQ. When the phantom was set up with a normal ventricular function (ejection fraction, 60\%) or decreased LVEF (ejection fraction, $45 \%$ or $33 \%$ ), there was a significant difference in EDV, ESV, and ejection fraction among 


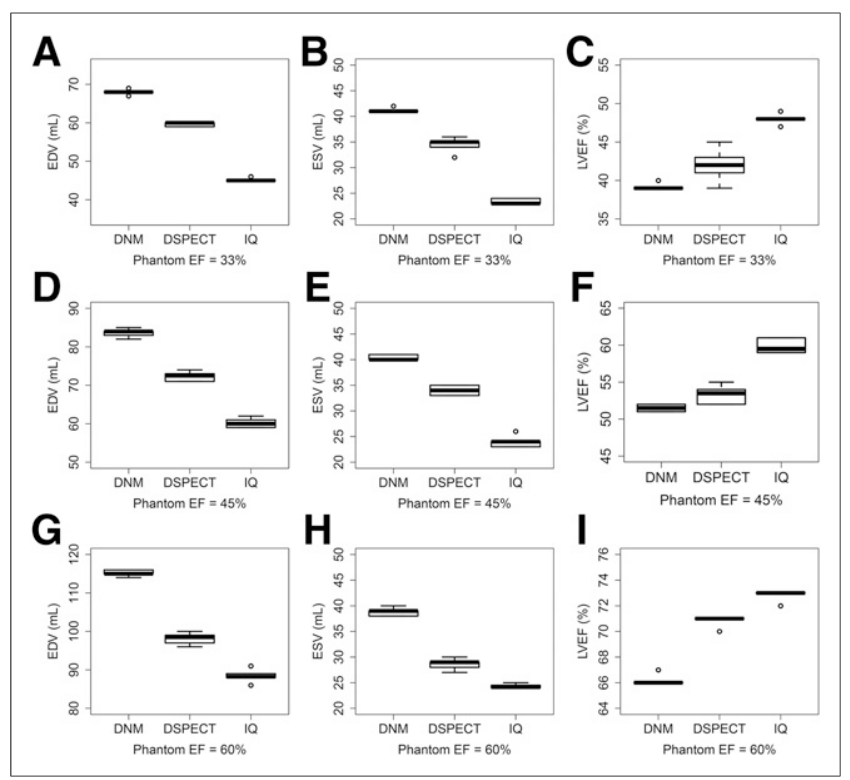

FIGURE 2. Phantom study comparison of mean EDV (A), mean ESV (B), and mean LVEF (C) for each camera and for LVEFs of $33 \%(A-C)$, $45 \%(\mathrm{D}-\mathrm{F})$, and $60 \%$ (G-I). Horizontal line of Tukey box marks median of samples; hinges of each box represent 25th and 75th percentiles. Error bars extending above and below each box show range of values that fall within 1.5 SDs of hinges. All $P<0.05$.

cameras (all $P<0.05$; Fig. 2). Count statistics had no impact on EDV, ESV, or ejection fraction, whatever the camera model.

\section{Clinical Data}

Global LV Function. Data obtained from 60 men (mean age, $57.7 \pm 10.9$ y) and 60 women (mean age, $63.1 \pm 13.3$ y) without evidence of heart disease were retrospectively assessed. Like the phantom data, the patient data showed that normal LV volume was higher for the DNM than for the D-SPECT or IQ. Indexed EDV $\left(\mathrm{mL} / \mathrm{m}^{2}\right)$ was $40.5 \pm 9.2$ vs. $37 \pm 7.9$ vs. $35.8 \pm 6.8$ (global $P$ value $<0.001)$, respectively, and indexed $\mathrm{ESV}\left(\mathrm{mL} / \mathrm{m}^{2}\right)$ was $12.5 \pm 5.3$ vs. $9.4 \pm 4.2$ vs. $8.3 \pm 4.4$ (global $P$ value $<0.001)$, respectively, resulting in a significantly decreased LVEF: $70.3 \% \pm$ $9.1 \%$ vs. $75.2 \% \pm 8.1 \%$ vs. $77.8 \% \pm 9.3 \%$ (global $P$ value $<$ 0.001), respectively. For indexed ESV, indexed EDV, and LVEF, multivariate ANOVA showed the sex and the camera type to have a significant effect $(P<0.05)$. Tracer $\left({ }^{99 \mathrm{~m} T c-t e t r o f o s m i n}\right.$ vs. ${ }^{99 \mathrm{~m} T c-s e s t a m i b i)}$ showed no statistically significant effect, and the clinical data from each tracer were therefore pooled for subsequent analyses. The results are presented in Figure 3, and the mean indexed EDV, indexed ESV, and LVEF obtained from each camera according to sex are shown in Table 1.

Segmental Wall Thickening and Motion. The assessment of segmental LV function yielded lower mean values for the DNM camera than for the D-SPECT or IQ. The respective mean values were $8.6 \pm 1.4 \mathrm{~mm}, 9.1 \pm 1.5 \mathrm{~mm}$, and $10.1 \pm 1.9 \mathrm{~mm}$ for motion (global $P<0.001$ ) and $49.1 \% \pm 12.2 \%, 56.9 \% \pm 13 \%$, and $58 \% \pm 15.1 \%$ for thickening (global $P<0.05$ ). The results are presented in Figure 4.

\section{DISCUSSION}

The phantom study demonstrated that estimated LV volume differed among the 3 cameras, especially for ESV, leading

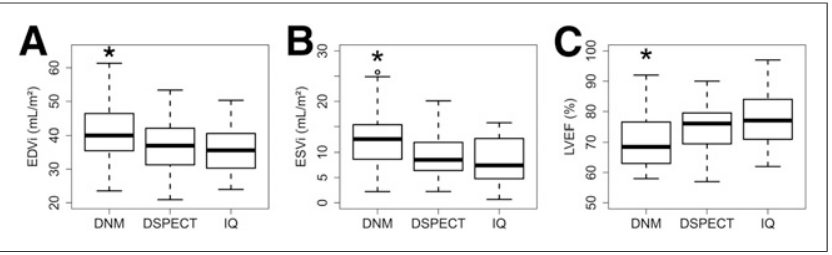

FIGURE 3. Patient study comparison of indexed EDV (A), indexed ESV (B), and LVEF (C) for each camera. Horizontal line in middle of Tukey box marks median of samples; hinges of each box represent 25th and 75th percentiles. Vertical lines extending above and below each box show range of values that fall within $1.5 \mathrm{SDs}$ of hinges. EDVi and $\mathrm{ESVi}=$ indexed EDV and ESV, respectively. ${ }^{{ }} P<0.05$ for DNM vs. D-SPECT and IQ.

to significant differences in normal and decreased LVEF. The estimated volume was higher when assessed using the CZT cameras, likely because of a better spatial resolution. The phantom study further indicated overestimations of normal LVEF, particularly for the IQ camera. In patients, the normal values of $L V$ function were dependent on the sex and the camera type.

\section{Global LV Function}

The effect of the type of camera on global and segmental function is likely related to spatial resolution. Imbert et al. (8) reported the following classification of measured central spatial resolution, in order of performance: DNM, $6.7 \mathrm{~mm}$; D-SPECT, $8.6 \mathrm{~mm}$; and IQ, $15.0 \mathrm{~mm}$. As shown in Figure 2, the results of our phantom study suggest that the LVEF overestimationbeing dramatically limited when the CZT cameras were used instead of the IQ-was a function of spatial resolution. The QGS algorithm used in this study works in 3-dimensional space and is also limited by spatial resolution. Germano et al., in an early study, found that a decrease in spatial resolution causes a reduction in estimated LV volume (21). When the end-systolic cavity size approaches the spatial resolution of the system, the partial-volume effect induces blurring at the myocardial wall edges and QGS miscalculates the endocardial surface, leading to a smaller estimated volume. Our findings also show that the increased spatial resolution of CZT-based cameras, by minimizing the partial-volume effect, results in increased volume measurements.

LV volume measurement may also be affected by reconstruction parameters (22), filtering and zooming (23), and absolute ventricular size (24). Clinical and simulation studies have previously reported an underestimation of LV volume, especially in small hearts $(23,25)$. Because the magnitude of underestimation depends on ventricle size, the relative effect is different between EDV and ESV calculations, leading to an overestimation of LVEF. Nakajima et al. (25) generated a set of mathematic simulations assuming an arbitrary resolution $(15.7,12.1,9.2,7.5$, and $6.9 \mathrm{~mm}$ in full width at half maximum). In that study, the authors demonstrated that the measured-to-true volume ratio was optimized by a higher resolution in numeric simulations. In addition, they also showed in a pediatric population that the use of a zoom during acquisition resulted in the same effect on volume assessment. These data suggest that factors favoring spatial resolution, such as reconstruction filters with a high cutoff frequency, high system resolution, and appropriate zooming, may improve gated SPECT quantification. 
TABLE 1

Clinical Results for Each Camera Model

\begin{tabular}{|c|c|c|c|c|c|c|}
\hline \multirow[b]{2}{*}{ Parameter } & \multicolumn{2}{|c|}{ DNM } & \multicolumn{2}{|c|}{ D-SPECT } & \multicolumn{2}{|c|}{$I Q$} \\
\hline & $\mathrm{F}$ & M & $\mathrm{F}$ & $M$ & $\mathrm{~F}$ & $M$ \\
\hline Age $(y)$ & $64.4 \pm 14.6(20-83)$ & $59.5 \pm 9.7(38-78)$ & $61.6 \pm 14.2^{\star}(31-82)$ & $51.6 \pm 10^{\dagger}(30-65)$ & $63.1 \pm 11.4(33-81)$ & $61.8 \pm 10.8^{\dagger}(37-78)$ \\
\hline BMI $\left(\mathrm{kg} / \mathrm{m}^{2}\right)$ & $27.8 \pm 5.7(19.5-42.5)$ & $27.1 \pm 3(22.5-32.4)$ & $31.1 \pm 8.3(19.5-45.9)$ & $28.5 \pm 5.9(21-45.9)$ & $28.4 \pm 7.8(18.7-50.7)$ & $31.8 \pm 7.1(18-43.8)$ \\
\hline $\mathrm{EDV}(\mathrm{mL})$ & $68 \pm 5.8^{\star}(43-100)$ & $86.5 \pm 20.2^{\S}(48-138)$ & $58.2 \pm 11.4^{\star}(41-83)$ & $82.5 \pm 10.7(62-105)$ & $62.9 \pm 14.2^{\star}(39-87)$ & $78.4 \pm 19.4^{\S}(50-115)$ \\
\hline $\operatorname{EDVi}\left(\mathrm{mL} / \mathrm{m}^{2}\right)$ & $38 \pm 8.6(23.8-56.1)$ & $43.1 \pm 9.3^{\S}(23.5-64.7)$ & $32.5 \pm 6.9^{\star}(20.9-46.8)$ & $41.5 \pm 6.2(30.5-53.3)$ & $35 \pm 6.1(26.1-45.9)$ & $38.2 \pm 9.3^{\S}(24-55.4)$ \\
\hline $\mathrm{ESV}(\mathrm{mL})$ & $18.2 \pm 8.8^{\star}(4-35)$ & $29.9 \pm 10.7^{\S}(15-58)$ & $12.9 \pm 6.2^{\star}(4-26)$ & $23.1 \pm 7.2(13-37)$ & $13.7 \pm 8.8(1-30)$ & $21.9 \pm 12.4^{\S}(5-55)$ \\
\hline ESVi $\left(\mathrm{mL} / \mathrm{m}^{2}\right)$ & $10.1 \pm 4.6^{\star}(2.2-19.3)$ & $14.9 \pm 5^{\S}(8.4-25.8)$ & $7.1 \pm 3.3^{\star}(2.2-13.2)$ & $11.6 \pm 3.8(7-20)$ & $7.4 \pm 4.5(1-15.7)$ & $10.6 \pm 6^{\S}(3-25.7)$ \\
\hline LVEF (\%) & $74.6 \pm 9.3^{\star}(58-92)$ & $65.9 \pm 6.5^{\ddagger \S}(58-78)$ & $78.5 \pm 7.6^{\star}(63-90)$ & $71.9 \pm 7.4^{\ddagger}(57-83)$ & $79.6 \pm 10(62-97)$ & $73.8 \pm 10.1^{\S}(52-92)$ \\
\hline Motion (mm) & $9 \pm 1.5^{\S}(6.7-11.9)$ & $8.3 \pm 1.1^{\S}(6.7-10.3)$ & $9.4 \pm 1.5(6.3-12.3)$ & $8.7 \pm 1.4(6.4-11.1)$ & $10.6 \pm 2.2^{\S}(7-16.6)$ & $9.4 \pm 1.7^{\S}(6.6-13.4)$ \\
\hline Thickening (\%) & $53.8 \pm 13.4^{\star}(35.8-79.1)$ & $44.4 \pm 8.9^{\S}(34.7-63.1)$ & $58.9 \pm 13.3(33-92.6)$ & $54.9 \pm 12.7^{\dagger}(35.2-79)$ & $62.2 \pm 17.3(36.9-113.1)$ & $51.9 \pm 12.8^{\dagger^{\S}}(32-81.1)$ \\
\hline $\begin{array}{l}{ }^{{ }^{*}} P<0.05 \text { vs. m } \\
{ }^{{ }} P<0.05 \text { D-SP } \\
{ }^{{ }} P<0.05 \text { DNM } \\
{ }^{\S_{P}}<0.05 \text { DNM } \\
\text { BMl }=\text { body mas } \\
\text { Data are mean } \pm\end{array}$ & $\begin{array}{l}\text { Iale sex using same camer } \\
\text { ECT vs. IQ within same se } \\
\text { vs. D-SPECT. } \\
\text { vs. IQ. } \\
\text { Ss index; EDVi = indexed } \\
\text { SD, followed by range in }\end{array}$ & $\begin{array}{l}\mathrm{EDV} ; \mathrm{ESVi}=\text { indexed ES } \\
\text { parentheses. }\end{array}$ & & & & \\
\hline
\end{tabular}

Sensitivity, contrast-to-noise ratio, photon scatter, and energy resolution may also affect assessments of volume and function. Previous phantom and clinical studies found a 3-fold improved sensitivity for the IQ camera, about a 4-fold improvement for the DNM, and nearly a 7-fold improvement for the D-SPECT, as well as demonstrating differences in sharpness profile measured in patient perfusion SPECT $(8,15)$. Photon scatter correction and compensation techniques based on energy windowing have been shown to improve image quality and volume measurements $(26,27)$. In particular, scattered photons in the main photopeak have a greater impact in smaller hearts than in larger hearts (28). Although CZT cameras offer a high energy resolution (15), the scatter fraction remains similar to that observed with conventional Anger cameras (18). Thus, it remains unclear whether the increased resolution contributes to the increased sharpness of images obtained with CZT cameras, an advantage that allows the myocardial edges to be detected easily and accurately, thus resulting in an increased ventricular volume compared with Anger cameras.

In patients, the assessment of LV volume and LVEF has been validated against cardiac MR $(12,13,29)$. Using the same software package for a DNM camera and a cardiac MR system, Giorgetti et al. (29) found an excellent correlation for both EDV $(r=0.92)$

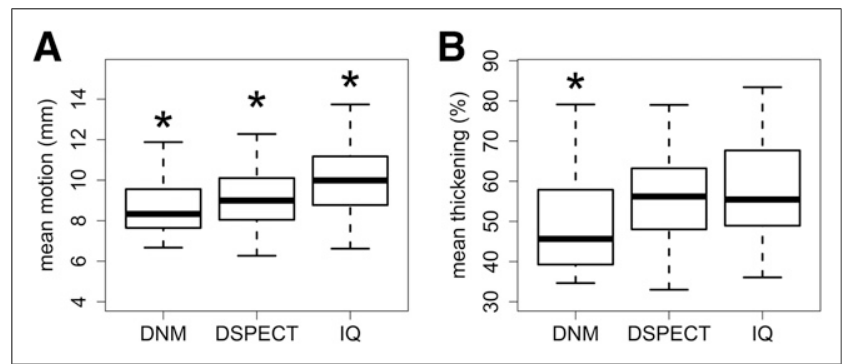

FIGURE 4. Comparison of mean wall motion $(A)$ and mean wall thickening (B) for each camera. ${ }^{*} P<0.05$ vs. other cameras. and ESV $(r=0.95)$, although there was a significant underestimation with the DNM camera (mean difference, $-33.2 \pm 26 \mathrm{~mL}$ for EDV and $-17.9 \pm 20 \mathrm{~mL}$ for ESV; $P<0.001$ ). For LVEF, not only was the correlation strong but the values were also similar $(49 \% \pm 16 \%$ vs. $51 \% \pm 15 \%$ for the DNM camera vs. cardiac MR; $r=0.91$ ). Cochet et al. (13) obtained similar results and further demonstrated that bias between the CZT camera and the cardiac MR system for the measurement of ESV (but not of EDV or LVEF) was greater in patients exhibiting moderate to severe defects. To our knowledge, there are no data comparing IQ to cardiac MR for the assessment of LV function. However, our study on patients without evidence of heart disease clearly showed that the CZT cameras yielded increased LV volume and decreased LVEF compared with the IQ camera. As previously documented $(30,31)$, sex-related differences were independent of camera type. These results agree with recent findings by Miao et al. (11), whose study on a healthy population found that in both men and women, indexed EDV and ESV were significantly higher for a DNM camera than for a dual-head conventional $\gamma$-camera. Our results further emphasize that the 2 commercially available CZT cameras provide different estimates of LV volume when assessed by means of QGS.

\section{Segmental LV Function}

Because of the partial-volume effect, a change in object size, as observed during myocardial contraction, results in changes in the apparent count density and is identified by a systolic increase in brightness. This method based on count density is widely used for assessing regional myocardial function with gated SPECT, but the exact quantification of the wall thickening remains limited (32). We previously demonstrated that the increased spatial resolution provided by CZT-based detectors may lead to an underestimation of regional myocardial wall thickening under hypertrophic conditions (12). In contrast, agreement with cardiac MR was stronger for myocardial wall motion than for wall thickening $(12,13)$. The present study demonstrated that regional myocardial 
wall thickening was decreased for the DNM, compared with the D-SPECT, whereas there was no significant difference in myocardial wall motion. These results support the measurement of wall motion by CZT cameras to assess segmental ventricular function.

\section{Study Limitations}

A first limitation of this study is that it was performed using poststress acquisitions and did not provide information on rest perfusion scans. The collected reference data in this retrospective study were from French centers that commonly use a stress-only protocol (33). In these centers, patients with a low likelihood of coronary artery disease and normal stress perfusion findings are discharged without undergoing the rest examination, resulting in a dramatically decreased radiation exposure. Consequently, this study could not assess the impact of camera type on transient ischemic dilation or myocardial stunning. Second, only a single reconstruction approach was used for each camera, and it is uncertain whether our findings apply to different reconstruction and filtering algorithms. Third, only one type of software was used, QGS. Methods based on statistical analysis of the distribution of count density would more significantly affect volume and ejection fraction measurements than methods, such as QGS, based on endocardial edge detection $(34,35)$. Therefore, the repeatability of our results using postprocessing methods avoiding edge detection is uncertain.

\section{CONCLUSION}

The different technical characteristics of the two commercially available CZT cameras and the conventional camera equipped with an astigmatic collimator resulted in different estimates of LV function when QGS was used for 3dimensional edge detection. These results confirm the need for sex- and camera-adjusted reference values for $\mathrm{LV}$ function parameters.

\section{DISCLOSURE}

The costs of publication of this article were defrayed in part by the payment of page charges. Therefore, and solely to indicate this fact, this article is hereby marked "advertisement" in accordance with 18 USC section 1734 . No potential conflict of interest relevant to this article was reported.

\section{ACKNOWLEDGMENTS}

We are grateful to Drs. Sylvie Petit, Mathilde Thelu, and Dimitri Bellevre and to the technologists at each institution (Lille, Toulouse, Nantes, Clermont-Ferrand, and Caen) for their help and technical assistance.

\section{REFERENCES}

1. Marcassa C, Bax JJ, Bengel F, et al. Clinical value, cost-effectiveness, and safety of myocardial perfusion scintigraphy: a position statement. Eur Heart J. 2008; 29:557-563.

2. Hachamovitch R, Berman DS, Kiat H, Cohen I, Friedman JD, Shaw LJ. Value of stress myocardial perfusion single photon emission computed tomography in patients with normal resting electrocardiograms: an evaluation of incremental prognostic value and cost-effectiveness. Circulation. 2002;105: $823-829$.
3. Thomas GS, Miyamoto MI, Morello AP, et al. Technetium 99m sestamibi myocardial perfusion imaging predicts clinical outcome in the community outpatient setting: the Nuclear Utility in the Community (NUC) Study. J Am Coll Cardiol. 2004;43:213-223

4. Ababneh AA, Sciacca RR, Kim B, Bergmann SR. Normal limits for left ventricular ejection fraction and volumes estimated with gated myocardial perfusion imaging in patients with normal exercise test results: influence of tracer, gender, and acquisition camera. J Nucl Cardiol. 2000;7: 661-668.

5. Sharir T, Slomka PJ, Hayes SW, et al. Multicenter trial of high-speed versus conventional single-photon emission computed tomography imaging: quantitative results of myocardial perfusion and left ventricular function. J Am Coll Cardiol. 2010;55:1965-1974.

6. Verger A, Imbert L, Yagdigul Y, et al. Factors affecting the myocardial activity acquired during exercise SPECT with a high-sensitivity cardiac CZT camera as compared with conventional Anger camera. Eur J Nucl Med Mol Imaging. 2014;41:522-528.

7. Duvall WL, Croft LB, Ginsberg ES, et al. Reduced isotope dose and imaging time with a high-efficiency CZT SPECT camera. J Nucl Cardiol. 2011;18:847857.

8. Imbert L, Poussier S, Franken PR, et al. Compared performance of high-sensitivity cameras dedicated to myocardial perfusion SPECT: a comprehensive analysis of phantom and human images. J Nucl Med. 2012;53:1897-1903.

9. Verger A, Djaballah W, Fourquet N, et al. Comparison between stress myocardial perfusion SPECT recorded with cadmium-zinc-telluride and Anger cameras in various study protocols. Eur J Nucl Med Mol Imaging. 2013;40: 331-340.

10. Sharir T, Ben-Haim S, Merzon K, et al. High-speed myocardial perfusion imaging initial clinical comparison with conventional dual detector anger camera imaging. JACC Cardiovasc Imaging. 2008;1:156-163.

11. Miao TL, Kansal V, Glenn Wells R, Ali I, Ruddy TD, Chow BJW. Adopting new gamma cameras and reconstruction algorithms: do we need to reestablish normal reference values? J Nucl Cardiol. August 25, 2015 [Epub ahead of print].

12. Bailliez A, Blaire T, Mouquet F, et al. Segmental and global left ventricular function assessment using gated SPECT with a semiconductor cadmium zinc telluride (CZT) camera: phantom study and clinical validation vs cardiac magnetic resonance. J Nucl Cardiol. 2014;21:712-722.

13. Cochet H, Bullier E, Gerbaud E, et al. Absolute quantification of left ventricular global and regional function at nuclear MPI using ultrafast CZT SPECT: initial validation versus cardiac MR. J Nucl Med. 2013;54:556-563.

14. Caobelli F, Kaiser SR, Thackeray JT, et al. IQ SPECT allows a significant reduction in administered dose and acquisition time for myocardial perfusion imaging: evidence from a phantom study. J Nucl Med. 2014; 55:2064-2070.

15. Bocher M, Blevis IM, Tsukerman L, Shrem Y, Kovalski G, Volokh L. A fast cardiac gamma camera with dynamic SPECT capabilities: design, system validation and future potential. Eur J Nucl Med Mol Imaging. 2010;37: 1887-1902.

16. Gambhir SS, Berman DS, Ziffer J, et al. A novel high-sensitivity rapidacquisition single-photon cardiac imaging camera. J Nucl Med. 2009;50:635643

17. Visser JJN, Sokole EB, Verberne HJ, et al. A realistic 3-D gated cardiac phantom for quality control of gated myocardial perfusion SPET: the Amsterdam gated (AGATE) cardiac phantom. Eur J Nucl Med Mol Imaging. 2004;31: 222-228.

18. Erlandsson K, Kacperski K, van Gramberg D, Hutton BF. Performance evaluation of D-SPECT: a novel SPECT system for nuclear cardiology. Phys Med Biol. 2009;54:2635-2649.

19. Cerqueira MD, Weissman NJ, Dilsizian V, et al. Standardized myocardial segmentation and nomenclature for tomographic imaging of the heart: a statement for healthcare professionals from the cardiac imaging committee of the council on clinical cardiology of the American Heart Association. Circulation. 2002;105: 539-542.

20. Shrout PE, Fleiss JL. Intraclass correlations: uses in assessing rater reliability. Psychol Bull. 1979;86:420-428.

21. Germano G, Kiat H, Kavanagh PB, et al. Automatic quantification of ejection fraction from gated myocardial perfusion SPECT. J Nucl Med. 1995;36:21382147.

22. Wright GA, McDade M, Martin W, Hutton I. Quantitative gated SPECT: the effect of reconstruction filter on calculated left ventricular ejection fractions and volumes. Phys Med Biol. 2002;47:N99-N105.

23. Véra P, Manrique A, Pontvianne V, Hitzel A, Koning R, Cribier A. Thalliumgated SPECT in patients with major myocardial infarction: effect of filtering 
and zooming in comparison with equilibrium radionuclide imaging and left ventriculography. J Nucl Med. 1999;40:513-521.

24. Nakajima K, Okuda K, Nyström K, et al. Improved quantification of small hearts for gated myocardial perfusion imaging. Eur J Nucl Med Mol Imaging. 2013;40: 1163-1170.

25. Nakajima K, Taki J, Higuchi T, et al. Gated SPET quantification of small hearts: mathematical simulation and clinical application. Eur J Nucl Med. 2000;27: 1372-1379.

26. Manrique A, Hitzel A, Véra P. Impact of photon energy recovery on the assessment of left ventricular volume using myocardial perfusion SPECT. $J$ Nucl Cardiol. 2004;11:312-317.

27. Galt JR, Cullom J, Garcia EV. Attenuation and scatter compensation in myocardial perfusion SPECT. Semin Nucl Med. 1999;29:204-220.

28. Hambye AS, Vervaet AM, Dobbeleir AA. Head-to-head comparison of uncorrected and scatter corrected, summed and end diastolic myocardial perfusion SPECT in coronary artery disease. Nucl Med Commun. 2004;25:347-353.

29. Giorgetti A, Masci PG, Marras G, et al. Gated SPECT evaluation of left ventricular function using a CZT camera and a fast low-dose clinical protocol: comparison to cardiac magnetic resonance imaging. Eur J Nucl Med Mol Imaging. 2013;40:1869-1875.
30. Sharir T, Kang X, Germano G, et al. Prognostic value of poststress left ventricular volume and ejection fraction by gated myocardial perfusion SPECT in women and men: gender-related differences in normal limits and outcomes. $J$ Nucl Cardiol. 2006;13:495-506.

31. Gebhard C, Stähli BE, Gebhard CE, et al. Gender- and age-related differences in rest and post-stress left ventricular cardiac function determined by gated SPECT. Int J Cardiovasc Imaging. 2014;30:1191-1199.

32. Danias PG, Ahlberg AW, Travin MI, et al. Visual assessment of left ventricular perfusion and function with electrocardiography-gated SPECT has high intraobserver and interobserver reproducibility among experienced nuclear cardiologists and cardiology trainees. J Nucl Cardiol. 2002;9:263-270.

33. Hesse B, Tägil K, Cuocolo A, et al. EANM/ESC procedural guidelines for myocardial perfusion imaging in nuclear cardiology. Eur J Nucl Med Mol Imaging. 2005;32:855-897.

34. Véra P, Koning R, Cribier A, Manrique A. Comparison of two three-dimensional gated SPECT methods with thallium in patients with large myocardial infarction. J Nucl Cardiol. 2000;7:312-319.

35. Feng B, Sitek A, Gullberg GT. Calculation of the left ventricular ejection fraction without edge detection: application to small hearts. J Nucl Med. 2002;43: 786-794. 